\title{
Role of $T$ cell immune response cDNA 7 on the pathology of acute graft-versus-host disease
}

\author{
FENG ZHU $^{1,2^{*}}$, YANQIU XU ${ }^{1 *}$, XIAOHUI FAN ${ }^{1}$, FAN ZHANG $^{1}$, DONG WANG $^{1}$, \\ JIANLIN QIAO ${ }^{1,3}$, SHENGYUN ZHU $^{1,3}$, KAI ZHAO $^{1,3}$, BIN PAN $^{1,3}$, CHONG CHEN $^{1,3}$, \\ LINGYU ZENG ${ }^{1,3}$, ZHENYU LI $^{1-3}$ and KAILIN XU ${ }^{1-3}$ \\ ${ }^{1}$ Blood Disease Institute, Xuzhou Medical University; ${ }^{2}$ Department of Hematology, The Affiliated Hospital of \\ Xuzhou Medical University; ${ }^{3}$ Key Laboratory of Bone Marrow Stem Cell, Xuzhou Medical University, \\ Xuzhou, Jiangsu 221002, P.R. China
}

Received September 7, 2019; Accepted August 18, 2020

DOI: $10.3892 / \mathrm{ol} .2020 .12163$

\begin{abstract}
Activation of T lymphocytes is the initiating factor of the occurrence of acute graft-versus-host disease (aGVHD), and cytotoxic T lymphocyte antigen-4 (CTLA-4) is the inhibitory receptor for activating $\mathrm{T}$ cells. $\mathrm{T}$ cell immune response cDNA 7 (TIRC7) is considered an upstream regulator of CTLA-4; however, little is understood regarding the effects of TIRC7 on the regulation of CTLA-4 in aGVHD. The purpose of the present study was to evaluate the regulatory effects of TIRC7 on aGVHD, mainly in the pathology. Recipient mice were exposed to a preconditioning dose of 7.5 Gy irradiation on the day of the transplantation and were divided into the following groups: Blank control group, bone marrow transplantation control group, total body irradiation group, mild-moderate aGVHD group and severe aGVHD group. According to the different administration of CTLA-4 and TIRC7 monoclonal antibodies, the mild-moderate and severe aGVHD groups were randomly divided into the hematopoietic stem cell transplantation (HSCT) and HSCT + CTLA-4/TIRC7 groups. Recipient mice were sacrificed at different time points post-HSCT for histopathological analysis by hematoxylin and eosin staining. Compared with the control and other experimental groups, the mice in the combined CTLA-4 and TIRC7 group exhibited ameliorated pathological injury, and lower pathology scores of the liver, lung and intestine. These data
\end{abstract}

Correspondence to: Professor Kailin Xu, Department of Hematology, The Affiliated Hospital of Xuzhou Medical University, 99 West Huaihai Road, Quanshan, Xuzhou, Jiangsu 221002, P.R. China

E-mail: lihmd@163.com

*Contributed equally

Key words: $\mathrm{T}$ cell immune response cDNA 7, cytotoxic $\mathrm{T}$ lymphocyte antigen-4, hematopoietic stem cell transplantation, acute graft-versus-host disease revealed that intraperitoneal injection of anti-TIRC7 and/or anti-CTLA-4 monoclonal antibody into mice could effectively alleviate the severity of aGVHD.

\section{Introduction}

Acute graft-versus-host disease (aGVHD) is a notable complication following allogeneic hematopoietic stem cell transplantation (allo-HSCT) (1). Abnormally activated T cells may be the initial factor for the occurrence of aGVHD (2). Activated $\mathrm{T}$ cells promote the secretion of cytokines, interferon- $\gamma($ IFN- $\gamma$ ) and interleukin-2 (IL-2), which can migrate to tissues, including in the lungs, liver and gut, and then result in organ dysfunction $(2,3)$. Therefore inhibition of $\mathrm{T}$ cell activation may effectively alleviate the incidence of aGVHD. Cytotoxic T lymphocyte antigen-4 (CTLA-4) is an inhibitory receptor following $\mathrm{T}$ cell activation. CTLA-4 negatively regulates $\mathrm{T}$ cell activity and deficient expression of CTLA-4 leads to autoimmune diseases $(4,5)$. It has been suggested that the expression of CTLA-4 is negatively associated with the severity of aGVHD (6). CTLA-4 can induce the phosphorylation of STAT3 and negatively modulate the proliferation and apoptosis of $\mathrm{CD}^{+} \mathrm{T}$ cells, as well as the induction of $\mathrm{T}$ helper (Th)1 cells (6). However, to the best of our knowledge, the molecule that acts upstream of CTLA-4 regulation in aGVHD has not been reported. As an upstream regulatory molecule of CTLA-4 (7-9), T cell immune response cDNA 7 (TIRC7), a seven transmembrane domain $\mathrm{G}$ protein, was first identified to play a role in graft rejection in 1998 (10). TIRC7 is expressed in immune organs, such as the spleen, and in $\mathrm{T}$ and $\mathrm{B}$ lymphocytes, playing a vital role in kidney and cardiac transplantation, as well as in collagen-induced arthritis $(8,9,11,12)$. Our previous study revealed that TIRC7 may be involved in the pathogenesis of immune thrombocytopenia (ITP) and may serve as an indicator for evaluating the efficacy of ITP treatment (13). In addition, TIRC7 plays a central role in allograft rejection and inflammation (14). Therefore, these findings demonstrated that TIRC7 is closely associated with the immune response. Further studies demonstrated that TIRC7 is essential in $\mathrm{T}$ cell activation $(15,16)$, and a specific antibody 
against TIRC7 can prevent immune activation via selective inhibition of Th1 and Th17 cell-associated cytokine expression of IL-2 and IFN- $\gamma$, indicating the critical role of TIRC7 in Th1 and Th17 cells $(7,17)$. In our previous study, increased TIRC7 expression was observed in patients with aGVHD (18). In addition, in lymphocytes obtained from TIRC7-deficient mice, the intracellular and cell surface expression of CTLA-4 was found to be markedly reduced compared with that in wild-type lymphocytes before and after activation (19). However, to the best of our knowledge, the mechanism by which TIRC7 influences the occurrence and development of aGVHD, and whether TIRC7 plays a regulatory role in aGVHD via CTLA-4 remain poorly understood. It was hypothesized that TIRC7 may positively regulate the function of CTLA-4 and inhibit T cell activation, thus attenuating the development and progression of aGVHD.

Although blockade of CTLA-4 signaling has previously been evaluated in GVHD, and early blocking of CTLA-4 signaling is expected to make $\mathrm{T}$ cells more aggressive and increase the severity of GVHD, as shown by Fevery et al (5) and Li et al (20), the findings of the present study are contradictory. Therefore, these conclusions are controversial.

In the present study, the role of TIRC7 in the regulation of aGVHD was investigated, and the liver, lung and intestine pathology were assessed. The results demonstrated that compared with the control and other experimental groups, the pathology scores of liver, lung and intestine of the aGVHD models in the group treated with a combination of CTLA-4 and TIRC7 monoclonal antibodies (mAbs) were the lowest.

\section{Materials and methods}

Materials. TIRC7 and CTLA-4 mAbs were custom generated by Wuhan GeneCreate Biological Engineering Co., Ltd., and the dilutions used of the anti-TIRC and anti-CTLA-4 mAbs were 0.25 and $0.40 \mathrm{mg} / \mathrm{ml}$ (dilution buffer, $0.01 \mathrm{mmol} / \mathrm{l} \mathrm{PBS}$ $\mathrm{pH} 7.4)$, respectively.

Animals. Specific-pathogen-free (SPF) male C57BL/6 mice (H-2K ${ }^{\text {b}}$; age, 8-12 weeks; weight, 23-28 g; 20 mice) were used as the donor mice and SPF female BALB/c mice $\left(\mathrm{H}-2 \mathrm{~K}^{\mathrm{d}}\right.$; age, 8-12 weeks; weight, 24-26 g; 220 mice) were used as the recipient mice. Recipient mice were sacrificed by cervical dislocation at days 7, 14, 21, 28 and 35 post-transplantation, and the endpoint of the animal experiments was day 40 post-transplantation, when the remaining mice were all sacrificed by cervical dislocation. The animals were purchased from Shanghai SLAC Laboratory Animal Co., Ltd., were housed in sterilized microisolator cages and were maintained in the individually ventilated cage room of the Experimental Animal Center of Xuzhou Medical University (Xuzhou, China). The temperature and relative humidity of the room were $19-21^{\circ} \mathrm{C}$ and $\sim 50 \%$, respectively. Animals were maintained on a 12:12-h light/dark cycle. Water was autoclaved, and feed was purchased from Shanghai SLAC Laboratory Animal Co. Ltd. Food and water were provided ad libitum. For the week prior to transplantation and the week following transplantation, the mice were provided autoclaved acidified water ( $\mathrm{pH} 2.5$ ) without any special requirements for feed.
After 2 weeks of acclimation to the laboratory conditions, the animal experiments commenced. All the procedures involving animals performed in the present study were in accordance with the Institutional Animal Care and Use Committee guidelines. The experimental protocols were approved by the Animal Ethics Committee of Xuzhou Medical University (Xuzhou, China).

Mouse model of aGVHD. The mouse model of aGVHD was established according to the previous articles published by our lab $(21,22)$. The recipient mice were exposed to a preconditioning dose of $7.5 \mathrm{~Gy}$ irradiation on the day of the transplantation $(0.66 \mathrm{~Gy} / \mathrm{min})$, and within $4 \mathrm{~h}$ post-irradiation, different numbers of bone marrow cells (5x10\% and splenic lymphocytes $\left(5 \times 10^{5} /\right.$ mouse for mild-moderate aGVHD groups; $5 \times 10^{6} /$ mouse for severe aGVHD groups) obtained from the donor mice were transfused into the recipient mice. C57BL/6 mice were sacrificed by cervical dislocation, immersed in iodine volts for $5 \mathrm{~min}$, and the tibia and femur were aseptically separated. After removing the attached muscles and fascia, the metaphysis was cut open. The bone marrow cavity was washed with PBS. Subsequently, a single cell suspension was produced by filtering through a 220-mesh stainless steel strainer. The bone marrow cells were prepared following centrifugation at $4^{\circ} \mathrm{C}$ and $800 \mathrm{x} \mathrm{g}$ for $5 \mathrm{~min}$ and suspended in PBS buffer. The spleen of C57BL/6 mice was separated and cut into small pieces of $5-10 \mathrm{~mm}^{3}$ and placed in a 200 -mesh stainless steel filter. Subsequently, the spleen was gently squeezed with the rubber tip of a sterile syringe core. After washing with the mouse lymphocyte separation solution (cat. no. DKW33-R0100; Dakewe Biotech Co., Ltd.) the cell suspension was prepared and transferred to the centrifuge tube. An equal volume of PBS buffer was slowly added and then the mixed liquor was centrifuged at room temperature at $800 \mathrm{x} \mathrm{g}$ for $20 \mathrm{~min}$. Subsequently, the buffy coat layer was aspirated, and 3-4 ml PBS buffer was added again (the centrifuge index was room temperature, $800 \mathrm{x} \mathrm{g}, 3 \mathrm{~min}$, twice). Finally, the splenic lymphocytes were suspended in PBS buffer. For the control groups, the BALB/c mice were randomly divided into the following three groups $(n=20$ mice per group): i) Blank control group [no total body irradiation (TBI), with normal saline infusion); ii) bone marrow transplantation (BMT) control group (after TBI, with bone marrow cells infusion, not splenic lymphocytes); and iii) TBI control group (only TBI without cell infusion). The mice in the TBI control group were all dead before day 15 post-transplantation, while the mice in the blank control group were all alive and there were 3 recipient mice in the BMT control group dead after transplantation (data not shown); therefore, in the results section, only the blank control group was presented as the control group. For experimental groups, preconditioned BALB/c mice were randomly divided into two groups ( $n=80$ per group): i) Group A, a BMT mouse model with mild-moderate aGVHD; and ii) group B, a BMT mouse model with severe aGVHD. The degree of aGVHD in the two groups was induced using different infusions of splenic lymphocytes, with $5 \times 10^{5} /$ mouse to induce mild-moderate aGVHD and $5 \times 10^{6} /$ mouse for severe aGVHD. Furthermore, according to the different administrations of CTLA-4 and 
Table I. Control groups of mice.

Control group ( $n=20$ per group)

Treatment

Blank control group

BMT control group

TBI control group
Mice were transfused physiological saline via the tail vein

Mice were transfused $5 \times 10^{6}$ donor bone marrow cells via the tail vein TBI only

BMT, bone marrow transplantation; TBI, total body irradiation.

Table II. Experimental groups of mice.

Treatment $^{\mathrm{a}}$

Experimental group

$(n=20$ per group)

Splenic lymphocytes

CTLA-4 ( $\mu \mathrm{g} /$ day)

TIRC7 ( $\mu \mathrm{g} /$ day)

\begin{tabular}{lrrr}
\hline A1 group & $5 \times 10^{5}$ & 0 & 0 \\
A2 group & $5 \times 10^{5}$ & 40 & 0 \\
A3 group & $5 \times 10^{5}$ & 0 & 25 \\
A4 group & $5 \times 10^{5}$ & 40 & 25 \\
B1 group & $5 \times 10^{6}$ & 0 & 0 \\
B2 group & $5 \times 10^{6}$ & 40 & 0 \\
B3 group & $5 \times 10^{6}$ & 0 & 25 \\
B4 group & $5 \times 10^{6}$ & 40 & 25 \\
\hline
\end{tabular}

${ }^{\mathrm{a}} \mathrm{All}$ groups were injected with $5 \times 10^{6}$ bone marrow cells. CTLA-4, cytotoxic T lymphocyte antigen- 4 ; TIRC7, T cell immune response cDNA 7 .

TIRC7 $\mathrm{mAbs}$, the $\mathrm{A}$ and $\mathrm{B}$ groups were randomly divided into four groups (A1-A4 and B1-B4 groups; $n=20$ mice per group). The CTLA-4 or TIRC7 mAbs were intraperitoneally injected into the mice. The optimal dosing time of CTLA-4 antibody was day 0 post-transplantation and its optimal dose was $40 \mu \mathrm{g} / \mathrm{mouse}$; the optimal dose and dosing times of TIRC7 antibody were $25 \mu \mathrm{g}$ /mouse and days $0,1,2,3$, 4 and 7 post-transplantation, respectively. Details of the groups are presented in Tables I and II.

Histopathological analyses. The health and behavior of the mice were monitored daily. Recipient mice were sacrificed by cervical dislocation at different time points [days 7, 14, 21, 28 and 35 post-transplantation according to the previous study (23)] during a total research period of 40 days post-transplantation, according to our previous study (22). A total of 3 mice per experimental group were sacrificed at each time point. Overall, 20 mice per group were used in order to prevent insufficient numbers of recipient mice due to the death of mice after transplantation for reasons other than aGVHD, and the remaining mice beyond the research period were sacrificed by cervical dislocation. Liver, lung and intestine tissue samples were then isolated. The tissues were fixed with formaldehyde solution at room temperature for $24-48 \mathrm{~h}$, dehydrated, embedded into paraffin at $60^{\circ} \mathrm{C}$ and sliced into $4-\mu \mathrm{m}$-thick sections using an RM2126 microtome (Leica Microsystems GmbH). The histological sections were then stained by hematoxylin and eosin staining at room temperature for 8-10 min and 4-5 sec, respectively. Next, the pathological changes were evaluated using a light microscope (Olympus Corporation; magnification, $\mathrm{x} 20$ ), and the number of slides examined per tissue sample was three. The images presented in Figs. 1-6 are representative of each tissue sample and were selected from the three slides examined per sample with the help from a pathologist.

Pathology scores following transplantation. Histopathological changes were examined with a light microscope (Olympus Corporation; magnification, x20) and the liver, lung and intestine tissue samples were scored using the aGVHD pathology score methods formulated by Blazar et al (24) and Kaplan et al (25). According to the degree of infiltration of inflammatory cells around blood vessels in the liver, lung and intestine, the pathology scores of each aGVHD model were determined, with a final score of 0-8 assigned per tissue sample. For example, intestinal GVHD was scored on the basis of crypt apoptosis $(0$, rare or none; 1 , occasional apoptotic bodies per 10 crypts; 2, few apoptotic bodies per 10 crypts; 3 , the majority of crypts contain an apoptotic body; 4 , the majority of crypts contain $>1$ apoptotic bodies) and inflammation ( 0 , none; 1 , mild; 2 , moderate; 3 , severe without ulceration; 4 , severe with ulceration), and these scores were added to obtain a final score of 0-8; so if the intestine had few apoptotic bodies per 10 crypts but severe inflammation without ulceration, the score was 5; if the intestine had the majority of crypts containing $>1$ apoptotic bodies and severe inflammation with ulceration, the score was 8 (25).

Statistical analysis. Data are presented as the mean \pm standard deviation. SPSS 16.0 software (SPSS, Inc.) was used 


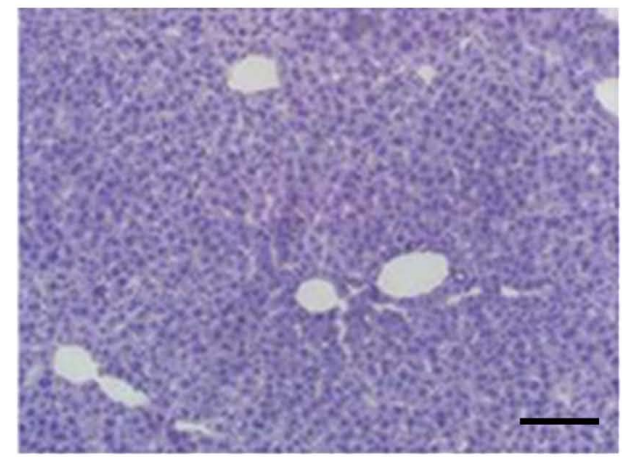

Control group

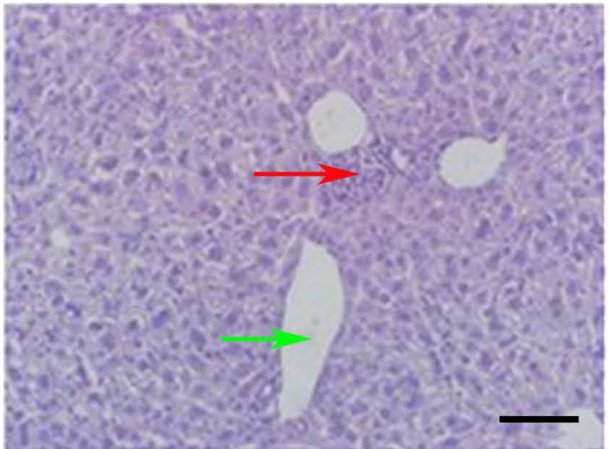

A1 group

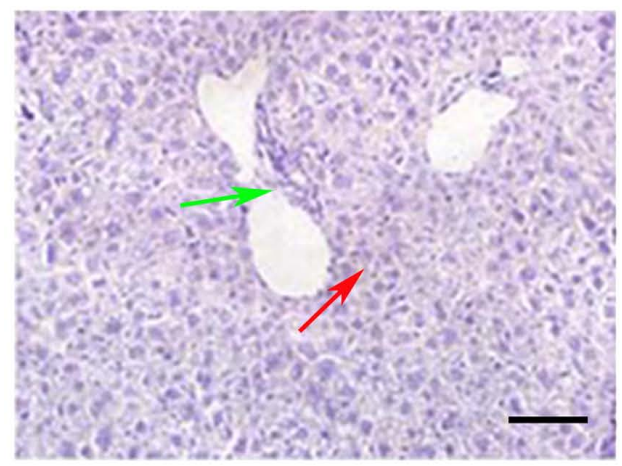

A3 group

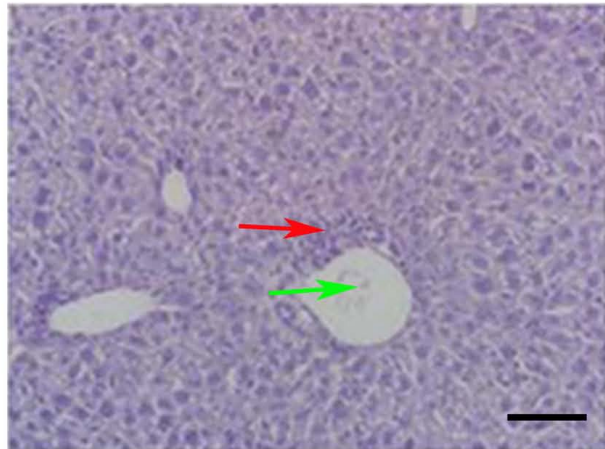

A2 group

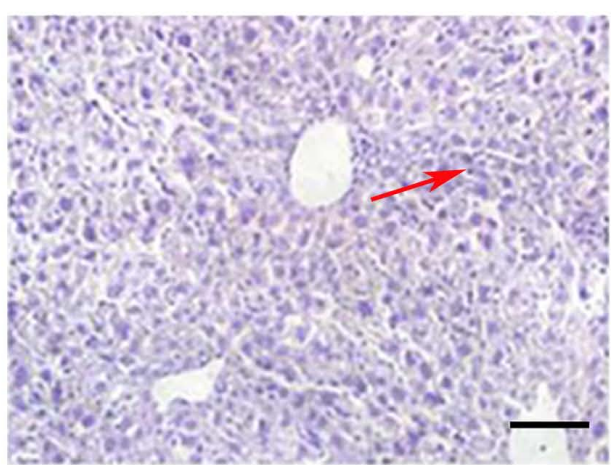

A4 group

Figure 1. Pathological changes of the liver in the control and A groups (magnification, $\mathrm{x} 20$ ) on day 21 post-allogeneic hematopoietic stem cell transplantation. A large number of lobulated nuclear cells and lymphocytes infiltrated the portal vein of the liver and local necrosis occurred (red arrows); in addition, hepatic sinusoids and central vein were dilated and blood stasis occurred (green arrows). Control group, blank control group; A1 group, mild-moderate aGVHD group; A2 group, mild-moderate aGVHD group with CTLA-4 intraperitoneal injection; A3 group, mild-moderate aGVHD group with TIRC7 intraperitoneal injection; A4 group, mild-moderate aGVHD group with CTLA-4 and TIRC7 intraperitoneal co-injection. Scale bar, $50 \mu \mathrm{m}$. aGVHD, acute graft-versus-host disease; TIRC7, T cell immune response cDNA 7; CTLA-4, cytotoxic T lymphocyte antigen-4.

for all statistical analyses. Kruskal-Wallis test and Dunn's test were employed to compare factors in different groups. $\mathrm{P}<0.05$ was considered to indicate a statistically significant difference.

\section{Results}

Pathological changes of aGVHD-targeted organs (liver, lung and intestine). As shown in Fig. 1, the manifestations of hepatic aGVHD were that a large number of lobulated nuclear cells and lymphocytes infiltrated the portal vein of the liver and local necrosis occurred; in addition, hepatic sinusoids and central vein were dilated and blood stasis occurred. The A4 group appeared to have fewer pathological changes in the liver compared with the other groups. The pathological changes of the lung and intestine at days 21 and 14 post-transplantation, respectively, are shown in Figs. 2 and 3. Inflammatory cells infiltrated around the blood vessels and part of the lung bronchiole structure was destroyed, and the intestinal mucosa exhibited partial shedding, and necrosis and inflammatory cell infiltration were observed. Figs. 4-6 demonstrated the pathological changes of liver, lung and intestine, respectively, in the blank control and B groups at different time points (days 21, 21 and 14, respectively) post-transplantation. The pathological changes in the liver in B groups (mainly B1-B3) included severe bile duct injury and lymphocyte infiltration (Fig. 4). Fig. 5 manifested that the lung bronchiole structure was destroyed and the inflammatory cells were infiltrated around the blood vessels. Fig. 6 exhibited that intestinal mucosa necrosis occurred in crypts and that most inflammatory cells were infiltrated. 


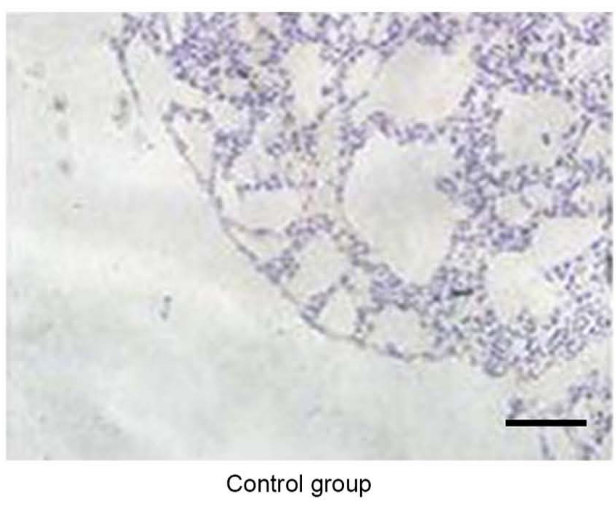

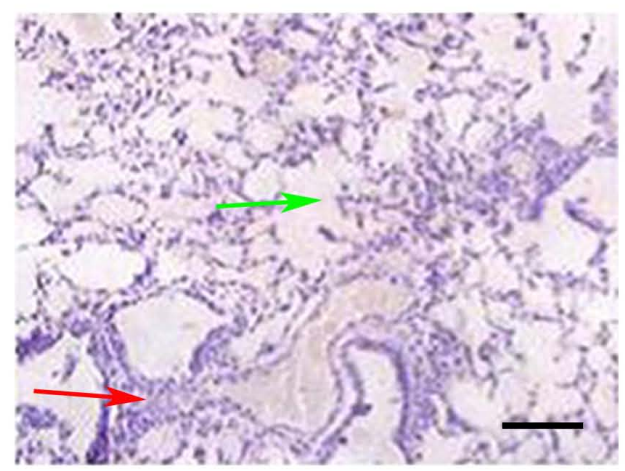

A1 group

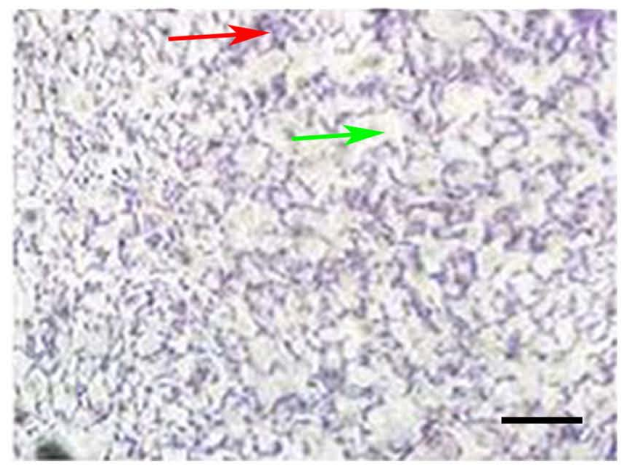

A3 group

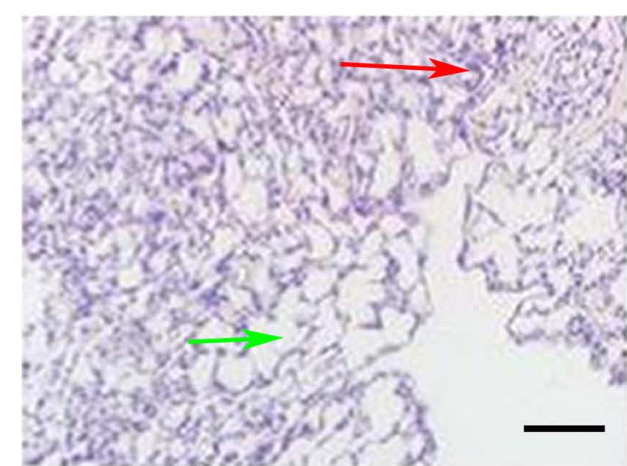

A2 group

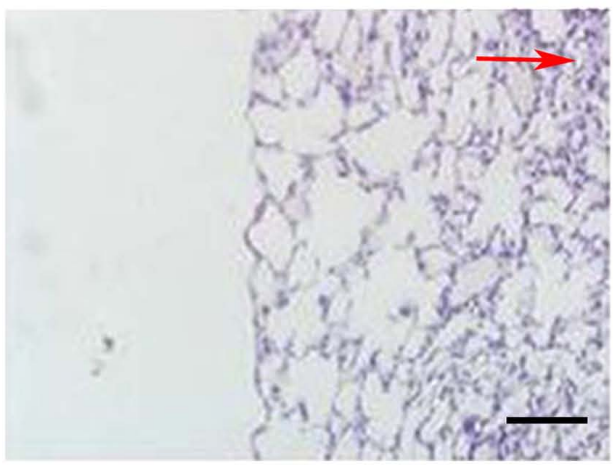

A4 group

Figure 2. Pathological changes of the lungs in the control and A groups (magnification, x20) on day 21 post-allogeneic hematopoietic stem cell transplantation. Inflammatory cells infiltrated around the blood vessels (red arrows) and part of the lung bronchiole structure was destroyed (green arrows). Control group, blank control group; A1 group, mild-moderate aGVHD group; A2 group, mild-moderate aGVHD group with CTLA-4 intraperitoneal injection; A3 group, mild-moderate aGVHD group with TIRC7 intraperitoneal injection; A4 group, mild-moderate aGVHD group with CTLA-4 and TIRC7 intraperitoneal co-injection. Scale bar, $50 \mu \mathrm{m}$. aGVHD, acute graft-versus-host disease; TIRC7, T cell immune response cDNA 7; CTLA-4, cytotoxic T lymphocyte antigen-4.

Pathological scores of aGVHD-targeted organs (liver, lung and intestine). As presented in Fig. 7, the pathological scores of liver in all experimental groups gradually increased until they peaked on day 21 post-transplantation, following which they gradually declined. Similar changes were observed in the lung and intestine, with a peak on days 21 and 14 post-transplantation, respectively (Figs. 8 and 9). From Figs. 7-9, it was observed that at days 14, 21, 28 and 35 post-transplantation, the pathological scores of liver, lung and intestine post-transplantation in the A4 group were significantly lower than those of the A1, A2 and A3 groups $(P<0.05)$, and the pathological scores of liver, lung and intestine in the A2 and A3 groups were also significantly lower than those of the A1 group $(\mathrm{P}<0.05)$. Similarly, at days 14 , 21,28 and 35 post-transplantation, the clinical scores of mice (liver, lung and intestine) among the B groups was lowest in group B4, and the scores in the B2 and B3 groups were also lower than those in the $\mathrm{B} 1$ group $(\mathrm{P}<0.05)$. By comparing the experimental groups, it was noted that the pathological scores of liver, lung and intestine in the A4 group were not significantly different from those in the B4 group on days 14, 21, 28 and 35 post-transplantation (data not shown). This suggested that there was no difference in the pathological changes between the mild-moderate and severe aGVHD groups after combined use of TIRC7 and CTLA-4 mAbs.

\section{Discussion}

At present, to the best of our knowledge, the pathogenesis of aGVHD remains unclear. Numerous studies have reported 


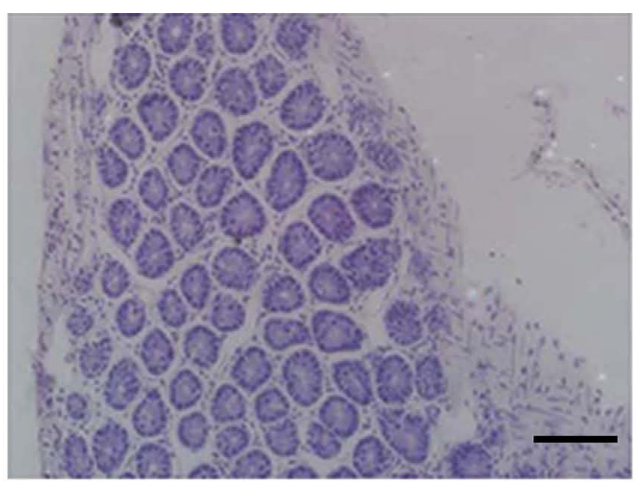

Control group

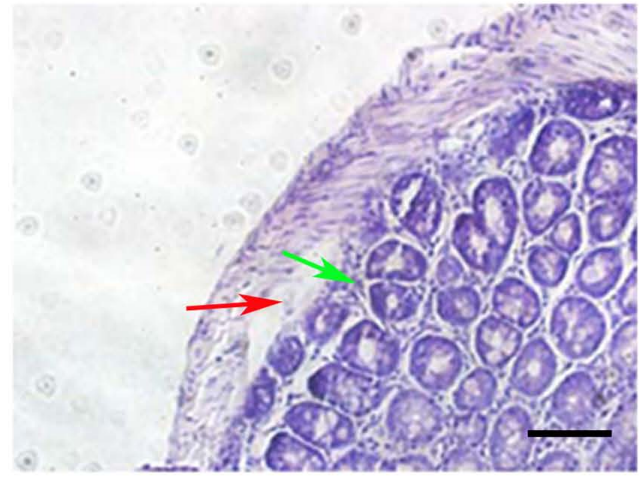

A1 group

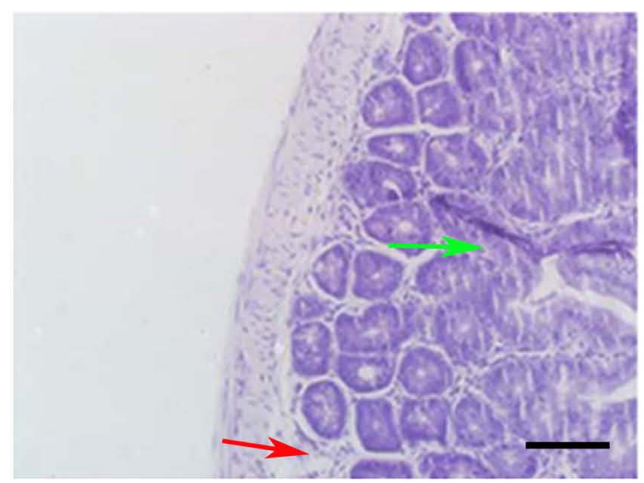

A3 group
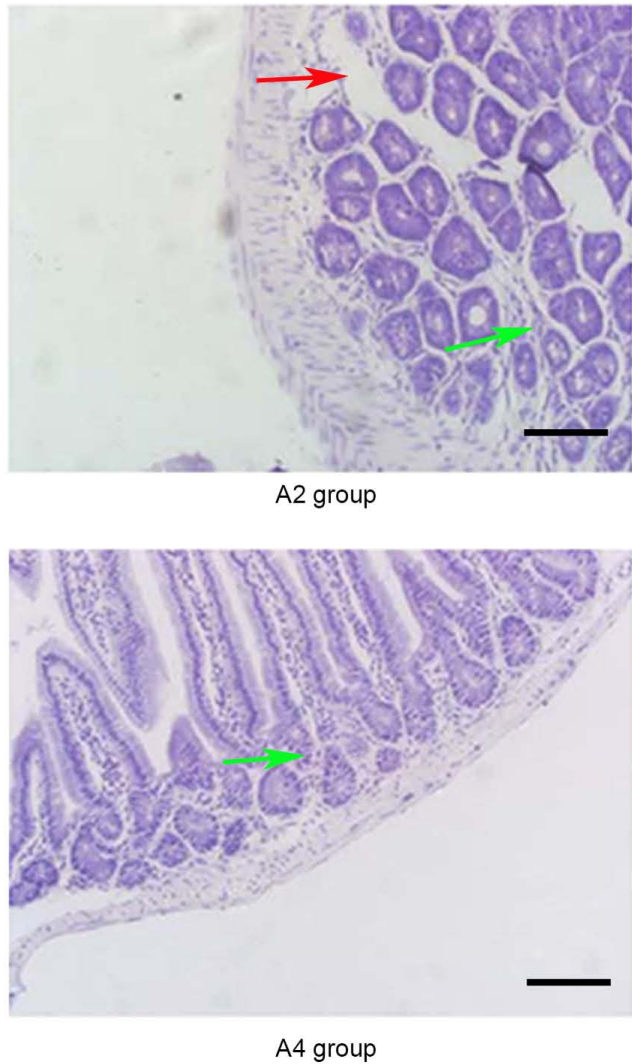

Figure 3. Pathological changes of the intestine in the control and A groups (magnification, x20) on day 14 post-allogeneic hematopoietic stem cell transplantation. The intestinal mucosa exhibited partial shedding (red arrows), and necrosis and inflammatory cell infiltration were observed (green arrows). Control group, blank control group; A1 group, mild-moderate aGVHD group; A2 group, mild-moderate aGVHD group with CTLA-4 intraperitoneal injection; A3 group, mild-moderate aGVHD group with TIRC7 intraperitoneal injection; A4 group, mild-moderate aGVHD group with CTLA-4 and TIRC7 intraperitoneal co-injection. Scale bar, $50 \mu \mathrm{m}$. aGVHD, acute graft-versus-host disease; TIRC7, T cell immune response cDNA 7; CTLA-4, cytotoxic T lymphocyte antigen-4.

that activation of $\mathrm{T}$ lymphocytes is the initial factor of aGVHD (2,26). A number of membrane proteins and their ligands have been found on the surface of $\mathrm{T}$ cells, which can be divided into three categories: i) The CD28 family, including CD28 and CTLA-4, the latter of which has homology with the CD28 molecule and binds to its ligand; ii) the costimulatory signal molecules associated with tumor necrosis factor and its receptor; and iii) the structural costimulatory factors associated with signaling lymphocyte activation molecules (27). A previous study has demonstrated that $\mathrm{T}$ cell activation can be enhanced by downregulating the expression of CTLA-4 following aGVHD (28). Yoo et al (29) found that the apoptosis of T cells is increased and the severity of aGVHD is decreased after overexpression of CTLA-4 in
T cells, indicating that CTLA-4 may play a negative regulatory role in aGVHD. Meanwhile, our previous study also found that CTLA-4 levels were decreased in patients with aGVHD before aGVHD treatment (corticosteroids as the first-line standard treatment for II-IV aGVHD) (18), whereas they were increased in patients with aGVHD after treatment (6). However, TIRC7, a novel membrane molecule, has been shown to be an essential molecule in the regulation of lymphocyte activation, and its expression is increased prior to aGVHD treatment (corticosteroids as the first-line standard treatment for II-IV aGVHD) but decreased following treatment $(6,18,30)$.

A previous study demonstrated that TIRC7 plays an important regulatory function in both $\mathrm{T}$ and $\mathrm{B}$ cell responses (19). 


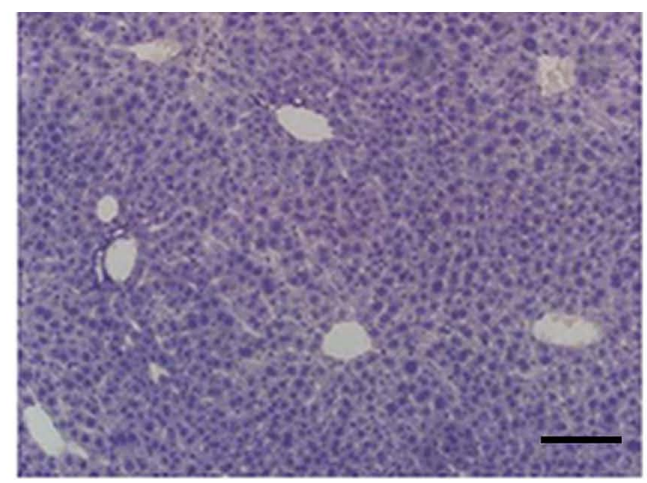

Control group

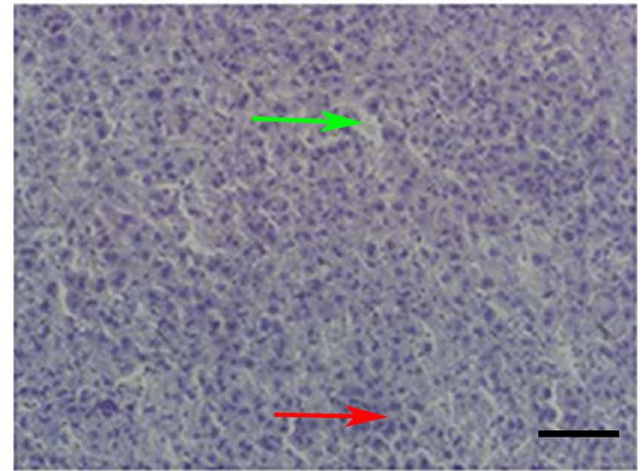

B1 group

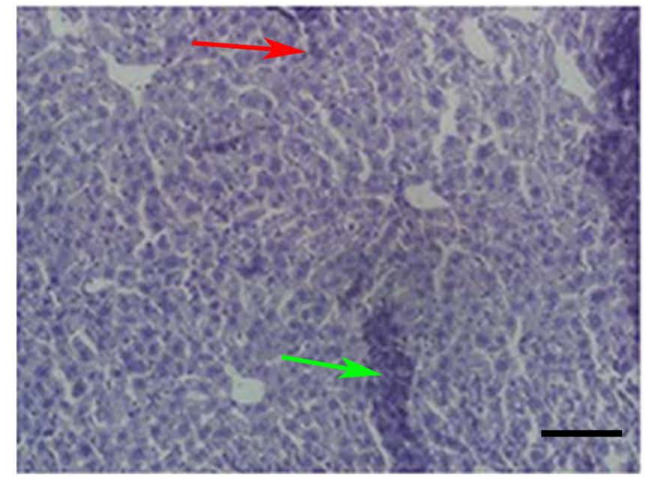

B3 group

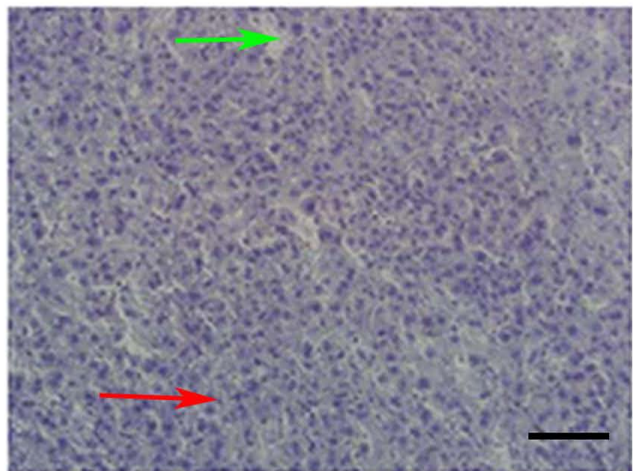

B2 group

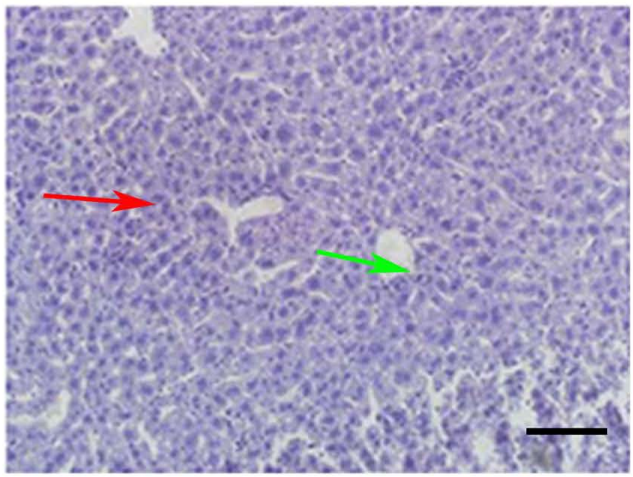

B4 group

Figure 4. Pathological changes of the liver in the control and B groups (magnification, x20) on day 21 post-allogeneic hematopoietic stem cell transplantation displaying severe bile duct injury (red arrows) and lymphocyte infiltration (green arrows). Control group, blank control group; B1 group, severe GVHD group; B2 group, severe GVHD group with CTLA-4 intraperitoneal injection; B3 group, severe GVHD group with TIRC7 intraperitoneal injection; B4 group, severe GVHD group with CTLA-4 and TIRC7 intraperitoneal co-injection. Scale bar, $50 \mu \mathrm{m}$. aGVHD, acute graft-versus-host disease; TIRC7, T cell immune response cDNA 7; CTLA-4, cytotoxic T lymphocyte antigen-4.

Meanwhile, as an upstream regulatory molecule of CTLA-4, TIRC7 inhibits $\mathrm{T}$ cell proliferation by modulation of CTLA-4 (16). Anti-TIRC7 mAb enhances the upregulation of CTLA-4 expression but suppresses the upregulation of CD25 in stimulated lymphocytes in vitro and in vivo (7). Antibodies against extracellular domains of TIRC7 prolonged allograft survival in rat and mouse transplantation models, and the prevention of rejection was mediated at least partially via induction of CTLA-4 in T cells (31). Our group found that the expression of CTLA-4 is negatively associated with the severity of aGVHD and inhibits Th1 cells by increasing STAT3 expression in aGVHD (6). Nevertheless, there is little knowledge regarding the exact mechanism by which TIRC7 is associated with aGVHD.
Based on these results, it was hypothesized that TIRC7 may inhibit $\mathrm{T}$ cell function via CTLA-4 to reduce the severity of aGVHD. Therefore, the present study evaluated and compared the effect of CTLA-4 and/or TIRC7 on the development of aGVHD in mice following HSCT and demonstrated that treatment with CTLA- 4 and TIRC7 mAbs could reduce the degree of the aGVHD.

According to the in vivo results, anti-CTLA- $4 \mathrm{mAb}$ administered by intraperitoneal injection could effectively alleviate the severity of aGVHD, which was consistent with a previous study that reported a novel mechanism able to effectively downregulate allogeneic $\mathrm{T}$ cell responses through deliberate ligation of CTLA-4 in vivo (32). Anti-TIRC7 mAb could also effectively mitigate the severity of aGVHD, which supports the 


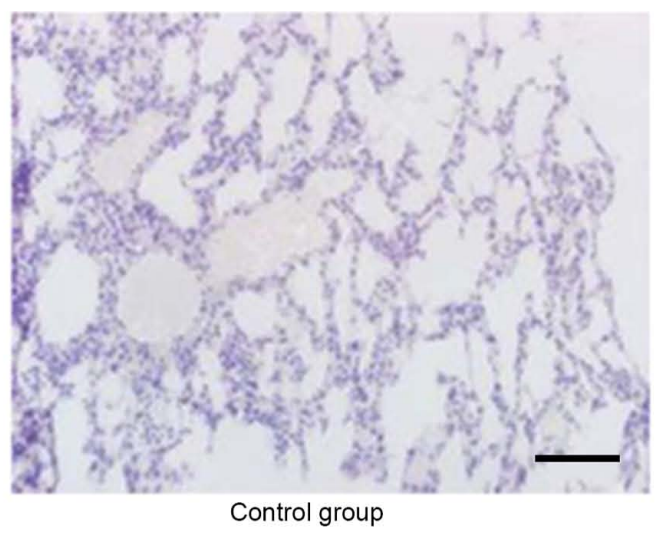

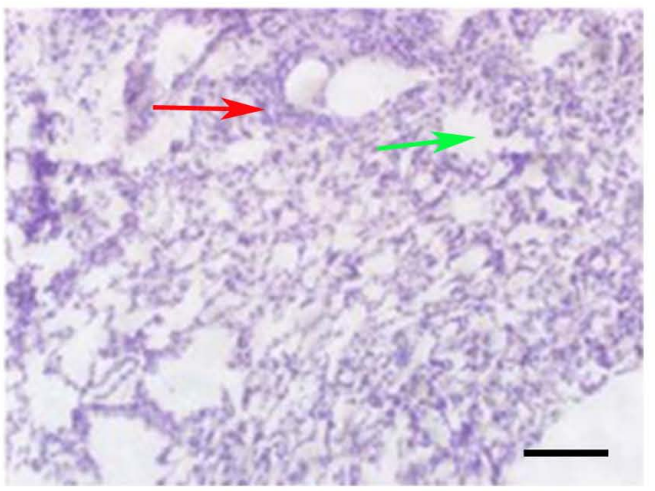

B1 group

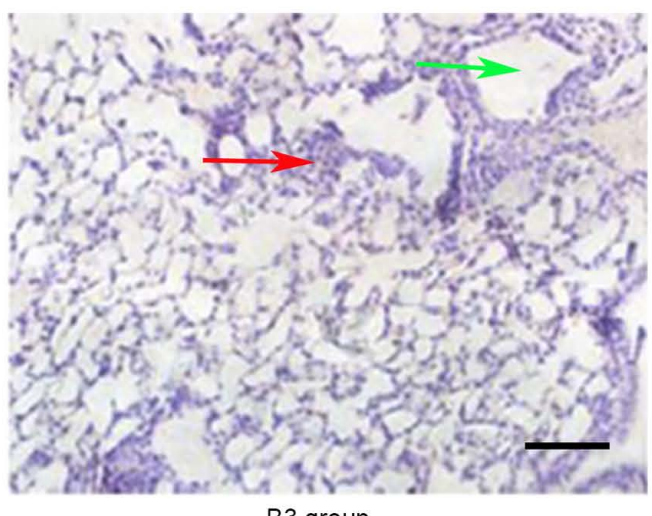

B3 group

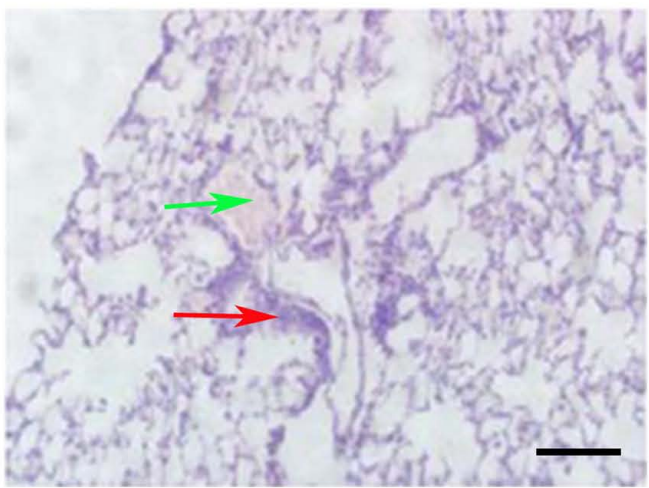

B2 group

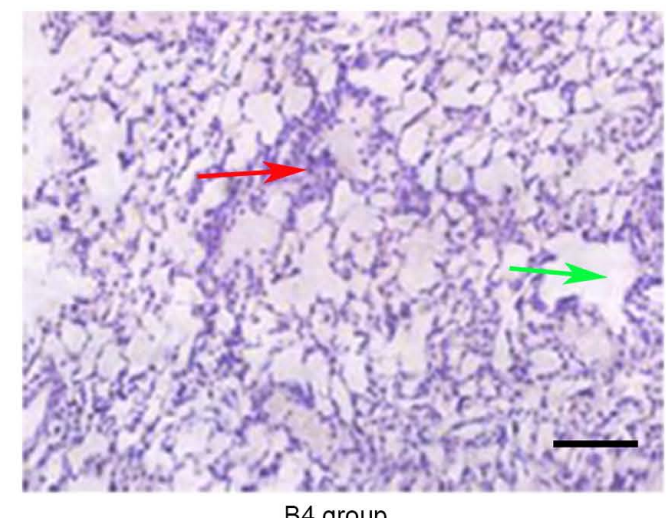

B4 group

Figure 5. Pathological changes of the lung in the control and B groups (magnification, x20) on day 21 post-allogeneic hematopoietic stem cell transplantation. The lung bronchiole structure was destroyed (red arrows) and inflammatory cells were infiltrated around the blood vessels (green arrows). Control group, blank control group; B1 group, severe GVHD group; B2 group, severe GVHD group with CTLA-4 intraperitoneal injection; B3 group, severe GVHD group with TIRC7 intraperitoneal injection; B4 group, severe GVHD group with CTLA-4 and TIRC7 intraperitoneal co-injection. Scale bar, $50 \mu \mathrm{m}$. aGVHD, acute graft-versus-host disease; TIRC7, $\mathrm{T}$ cell immune response cDNA 7; CTLA-4, cytotoxic T lymphocyte antigen-4.

data published by Kumamoto et al (7), which demonstrated that targeting TIRC7 with anti-TIRC7 mAb diminishes lymphocyte infiltration into grafts and delays morphological graft damage. Notably, combined administration of anti-TIRC7 and anti-CTLA-4 antibodies had an additive effect in the present study. The optimal doses of TIRC7 and CTLA-4 mAbs to treat aGVHD in mouse models have not yet been reported. Referring to the available literature $(7,33)$ and our preliminary results (data not shown), the optimal administration time, dose and route of CTLA- $4 \mathrm{mAb}$ were determined to be at day 0 within $4 \mathrm{~h}$ after irradiation, $40 \mu \mathrm{g}$ per mouse and intraperitoneal injection, respectively. The optimal administration time, dose and route of TIRC $7 \mathrm{mAb}$ were determined to be days $0,1,3,4$ and 7 post-irradiation, $25 \mu \mathrm{g}$ per mouse and intraperitoneal injection, respectively. It was hypothesized that intraperitoneal injection of both TIRC7 and CTLA-4 mAbs into recipient mice could activate the immunoreceptor tyrosine-based inhibitory motif of the intracellular domain of two protein molecules, negatively regulating the function of $\mathrm{T}$ lymphocytes, and thereby effectively mitigating the severity of aGVHD. In the present study, TIRC7 and CTLA-4 mAbs exhibited an additive effect and the combined use could reduce the degree of aGVHD more effectively compared with TIRC7 or CTLA-4 mAb alone. Due to the focus of the current study on the aspect of pathology, the data of the cytokine expression were not presented; however, compared with the control group, increased levels of IFN- $\gamma$, 


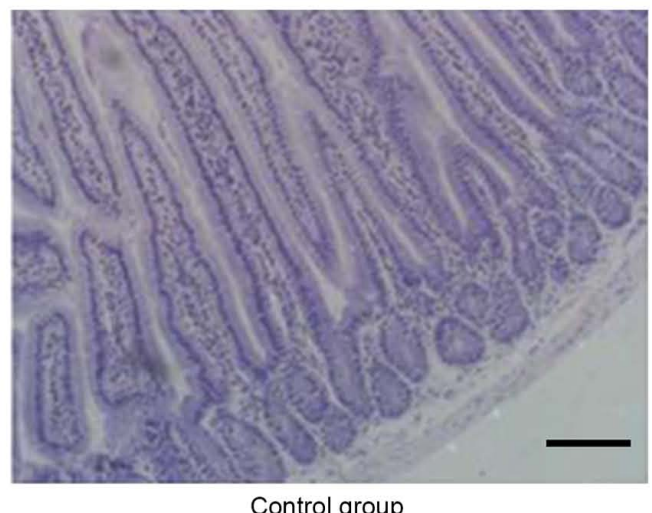

Control group

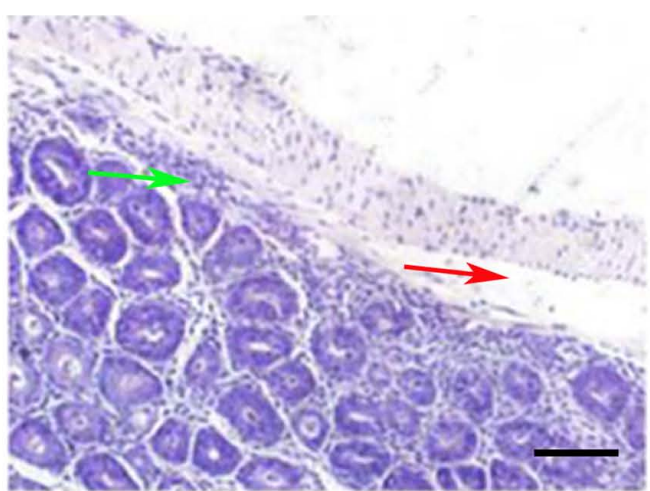

B1 group

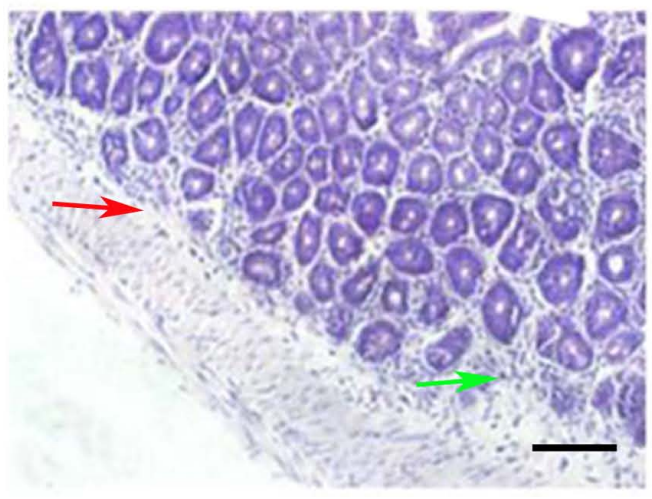

B3 group

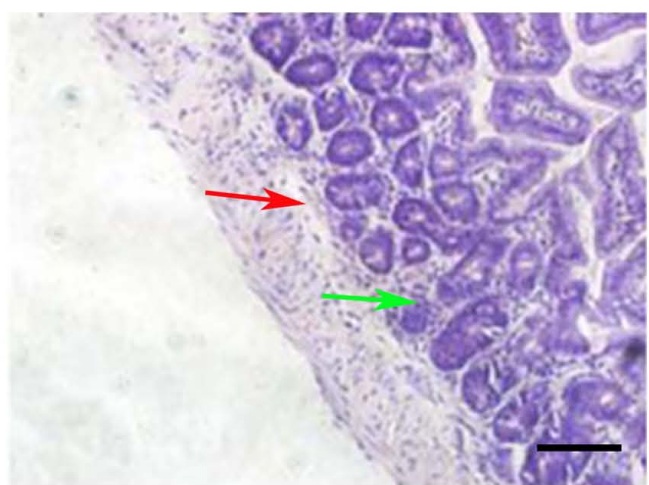

B2 group

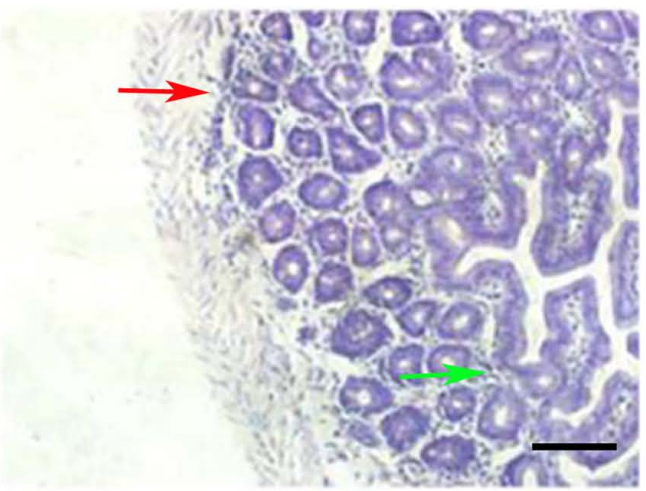

B4 group

Figure 6. Pathological changes of the intestine in the control and B groups (magnification, x20) on day 14 post-allogeneic hematopoietic stem cell transplantation. Intestinal mucosa necrosis occurred in crypts (red arrows) and most inflammatory cells were infiltrated (green arrows). Control group, blank control group; B1 group, severe GVHD group; B2 group, severe GVHD group with CTLA-4 intraperitoneal injection; B3 group, severe GVHD group with TIRC7 intraperitoneal injection; B4 group, severe GVHD group with CTLA-4 and TIRC7 intraperitoneal co-injection. Scale bar, $50 \mu$ m. aGVHD, acute graft-versus-host disease; TIRC7, T cell immune response cDNA 7; CTLA-4, cytotoxic T lymphocyte antigen-4.

IL-17 and IL-22, and decreased IL-4 levels were observed in groups A and B, indicating an imbalance of Th1/17/22 and Th2 cells in the pathogenesis of aGVHD (data not shown). The histopathological scores of mice with aGVHD in the experimental group supported this view. Notably, the degree of aGVHD manifestation in the mild-moderate aGVHD group was less, for example, the aGVHD scores of the liver, lung and intestine were lower compared with those in the severe aGVHD group; however, there were no differences in pathological changes between the mild-moderate and severe GVHD groups after combined use of TIRC7 and CTLA-4 mAbs (data not shown). The present results provide a preliminary theoretical basis for the prevention and treatment of aGVHD in patients with allo-HSCT by using TIRC7 and CTLA-4 mAbs in the future. TIRC7 and CTLA-4 mAbs could reduce the severity of aGVHD in mice; however, the current pathogenesis of whether the two mAbs have a synergistic effect in aGVHD is not yet clear and needs to be further explored in future studies.

In conclusion, TIRC7 may positively regulate the function of CTLA-4, inhibit $\mathrm{T}$ cell activation and the secretion of cytokines, and downregulate the progression of aGVHD. Furthermore, combined administration of CTLA-4 and TIRC7 mAbs could reduce GVHD more effectively in mouse models, which suggests that their combined application may be a novel therapy for preventing and treating GVHD. 


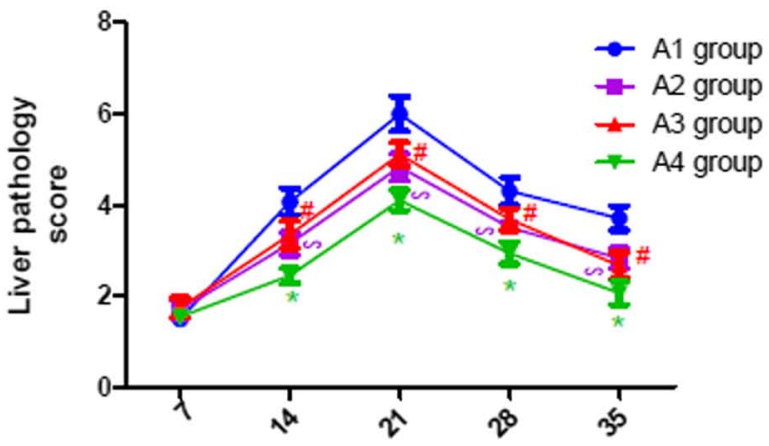

Days post-allo-HSCT

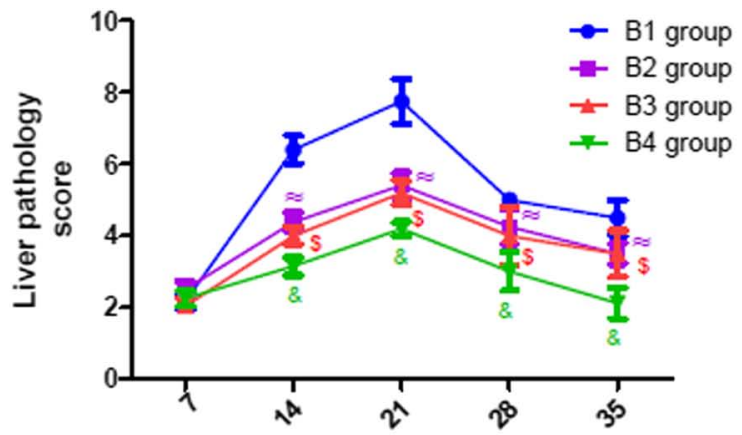

Days post-allo-HSCT

Figure 7. Liver pathology scores of mice with acute graft-versus-host disease post-allogeneic hematopoietic stem cell transplantation (n=3). ${ }^{*} \mathrm{P}<0.05 \mathrm{vs}$. A1/A2/A3 groups; ${ }^{\#} \mathrm{P}<0.05$ vs. A1 group; ${ }^{\circ} \mathrm{P}<0.05$ vs. A1 group; ${ }^{\circledR} \mathrm{P}<0.05$ vs. B1/B2/B3 groups; ${ }^{\mathrm{P}} \mathrm{P}<0.05$ vs. B1 group; ${ }^{\sim} \mathrm{P}<0.05$ vs. B1 group. allo-HSCT, allogeneic hematopoietic stem cell transplantation.

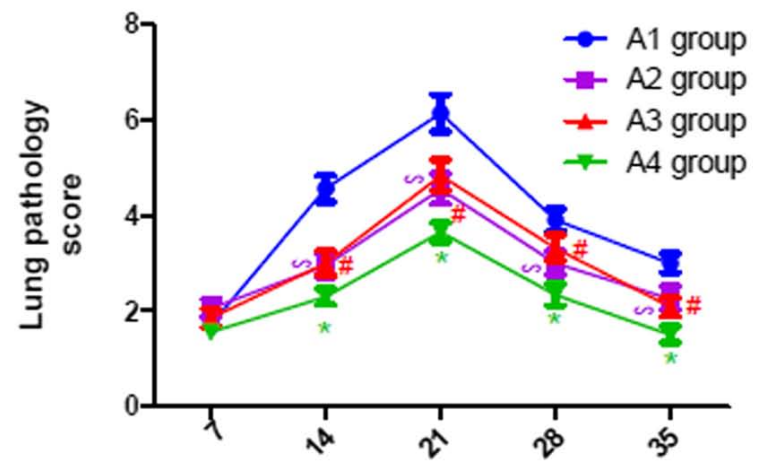

Days post-allo-HSCT

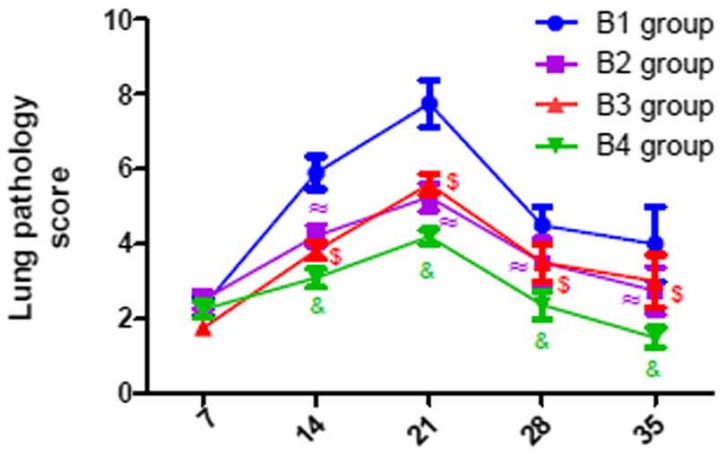

Days post-allo-HSCT

Figure 8. Lung pathology scores of mice with acute graft-versus-host disease post-allogeneic hematopoietic stem cell transplantation (n=3). ${ }^{*} \mathrm{P}<0.05 \mathrm{vs}$. A1/A2/A3 groups; ${ }^{\text {P }}<0.05$ vs. A1 group; ${ }^{\infty} \mathrm{P}<0.05$ vs. A1 group; ${ }^{\circledR} \mathrm{P}<0.05$ vs. B1/B2/B3 groups; ${ }^{\mathrm{P}} \mathrm{P}<0.05$ vs. $\mathrm{B} 1$ group; $~{ }^{\mathrm{P}}<0.05$ vs. B1 group. allo-HSCT, allogeneic hematopoietic stem cell transplantation.

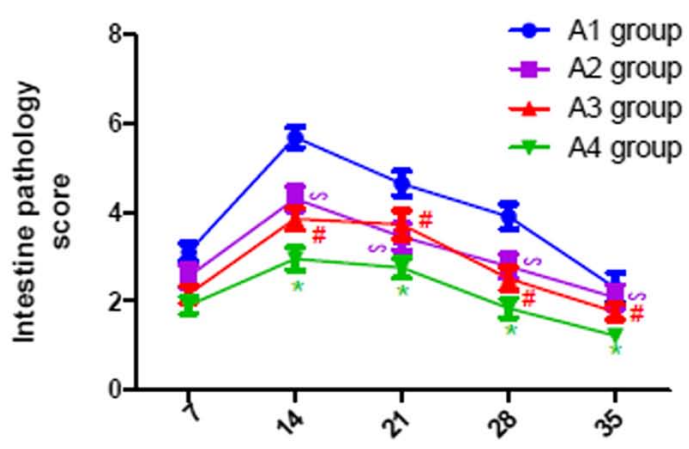

Days post-allo-HSCT

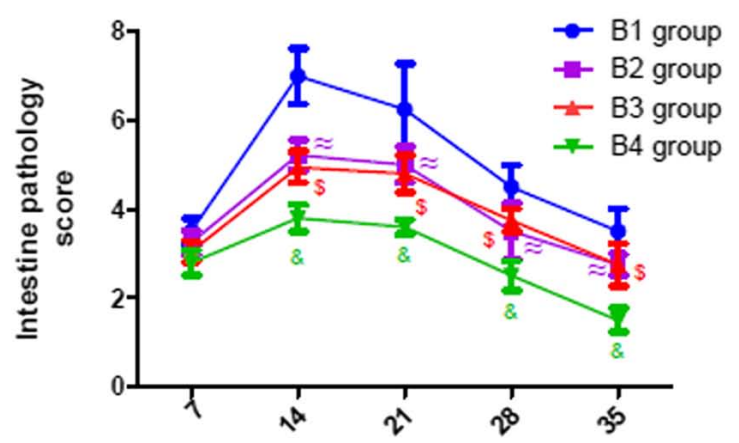

Days post-allo-HSCT

Figure 9. Intestine pathology scores of mice with acute graft-versus-host disease post-allogeneic hematopoietic stem cell transplantation ( $\mathrm{n}=3$ ). ${ }^{*} \mathrm{P}<0.05$ vs. A1/A2/A 3 groups; ${ }^{\#} \mathrm{P}<0.05$ vs. A1 group; ${ }^{\infty} \mathrm{P}<0.05$ vs. A1 group; ${ }^{\circledR} \mathrm{P}<0.05$ vs. B1/B2/B3 groups; ${ }^{\$} \mathrm{P}<0.05$ vs. $\mathrm{B} 1$ group; $\approx \mathrm{P}<0.05$ vs. $\mathrm{B} 1$ group. allo-HSCT, allogeneic hematopoietic stem cell transplantation.

\section{Acknowledgements}

The authors would like to thank Miss Xiangbu Wang (Department of Pathology, The Affiliated Hospital of Xuzhou Medical University, Xuzhou, China) for her assistance in the selection of the pathological images of the present study.

\section{Funding}

This study was supported by the National Natural Science Foundation of China (grant nos. 81270637, 81300377 and 81600145), the Natural Science Foundation of Jiangsu Province (grant nos. BK20160226 and BK20160232), the China 
Postdoctoral Science Foundation (grant no. 2016M590508), the Foundation of Jiangsu Province Six Talents Peak (grant no. 2015-wsw-058) and the Foundation of Jiangsu Province Six-one Project (grant no. LGY2018084).

\section{Availability of data and materials}

The datasets used and/or analyzed during the current study are available from the corresponding author on reasonable request.

\section{Authors' contributions}

FZhu, YX and XF designed the experiments and wrote the first draft of the manuscript. FZha and DW primarily performed the experiments, wrote the manuscript and prepared the figures. JQ and SZ were involved in performing the experiments. KZ, $\mathrm{BP}$ and $\mathrm{CC}$ made substantial contributions to the acquisition and analysis of data. LZ, ZL and KX made contributions to the analysis and interpretation of data and revised the manuscript. All authors read and approved the final manuscript.

\section{Ethics approval and consent to participate}

All the procedures involving animals performed in the present study were in accordance with the Institutional Animal Care and Use Committee guidelines. The experimental protocols were approved by the Animal Ethics Committee of Xuzhou Medical University (Xuzhou, China).

\section{Patient consent for publication}

Not applicable.

\section{Competing interests}

The authors declare that they have no competing interests.

\section{References}

1. Ammer J, Prantl L, Holler B, Holler K, Landfried D, Wolff S, Karrer R, Andreesen R and Holler E: Successful treatment of a refractory skin ulcer in chronic cutaneous GvHD after allogeneic HSCT with split-thickness skin allografting from the stem cell donor. Bone Marrow Transplant 47: 1368-1369, 2002.

2. Ferrara JL, Levine JE, Reddy P and Holler E: Graft-versus-host disease. Lancet 373: 1550-1561, 2009.

3. Lai HY, Chou TY, Tzeng CH and Lee OK: Cytokine profiles in various graft-versus-host disease target organs following hematopoietic stem cell transplantation. Cell Transplant 21: 2033-2045, 2012.

4. Tivol EA, Borriello F, Schweitzer AN, Lynch WP, Bluestone JA and Sharpe AH: Loss of CTLA-4 leads to massive lymphoproliferation and fatal multiorgan tissue destruction, revealing a critical negative regulatory role of CTLA-4. Immunity 3 : 541-547, 1995.

5. Fevery S, Billiau AD, Sprangers B, Rutgeerts O, Lenaerts C, Goebels J, Landuyt W, Kasran A, Boon L, Sagaert X, et al: CTLA-4 blockade in murine bone marrow chimeras induces a host-derived antileukemic effect without graft-versus-host disease. Leukemia 21: 1451-1459, 2007.

6. Zhu F, Zhong XM, Qiao J, Liu Q, Sun HY, Chen W, Zhao K, Wu QY, Cao J, Sang W, et al: Cytotoxic T Lymphocyte Antigen-4 Down-Regulates T Helper 1 cells by increasing expression of signal transducer and activator of transcription 3 in Acute Graft-versus-Host Disease. Biol Blood Marrow Transplant 22: 212-219, 2016
7. Kumamoto Y, Tomschegg A, Bennai-Sanfourche F, Boerner A, Kaser A, Schmidt-Knosalla I, Heinemann T, Schlawinsky M, Blumberg RS, Volk HD and Utku N: Monoclonal antibody specific for TIRC7 induces donor-specific anergy and prevents rejection of cardiac allografts in mice. Am J Transplant 4: 505-514, 2004.

8. Morgun A, Shulzhenko N, Diniz RV, Almeida DR, Carvalho AC and Gerbase-DeLima M: Cytokine and TIRC7 mRNA expression during acute rejection in cardiac allograft recipients. Transplant Proc 33: 1610-1611, 2001.

9. Kumamoto Y, Tamura A, Volk HD, Reinke P, Lohler J, Tullius SG and Utku N: TIRC7 is induced in rejected human kidneys and anti-TIRC7 mAb with FK506 prolongs survival of kidney allografts in rats. Transpl Immunol 16: 238-244, 2006.

10. Utku N, Heinemann T, Tullius SG, Bulwin GC, Beinke S, Blumberg RS, Beato F, Randall J, Kojima R, Busconi L, et al: Prevention of acute allograft rejection by antibody targeting of TIRC7, a novel T cell membrane protein. Immunity 9: 509-518, 1998.

11. Utku N, Heinemann T, Winter M, Bulwin GC, Schlawinsky M, Fraser P, Nieuwenhuis EE, Volk HD and Blumberg RS: Antibody targeting of TIRC7 results in significant therapeutic effects on collagen-induced arthritis in mice. Clin Exp Immunol 144: 142-151, 2006.

12. Shulzhenko N, Morgun A, Rampim GF, Franco M, Almeida DR, Diniz RV, Carvalho AC and Gerbase-DeLima M: Monitoring of intragraft and peripheral blood TIRC7 expression as a diagnostic tool for acute cardiac rejection in humans. Hum Immunol 62: 342-347, 2001.

13. Zhu F, Qiao JL, Wu QY, Cao J, Zeng LY, Li ZY and Xu KL: Elevated levels of T-cell immune response cDNA 7 in patients with immune thrombocytopenia. Hematology 19: 477-482, 2014

14. Utku N, Heinemann T and Milford EL: T-cell immune response cDNA 7 in allograft rejection and inflammation. Curr Opin Investig Drugs 8: 401-410, 2007.

15. Heinemann T, Bulwin GC, Randall J, Schnieders B, Schnieders K, Volk HD, Milford E, Gullans SR and Utku N: Genomic organization of the gene coding for TIRC7, a novel membrane protein essential for T cell activation. Genomics 57: 398-406, 1999.

16. Bulwin GC, Heinemann T, Bugge V, Winter M, Lohan A Schlawinsky M, Schulze A, Walter S, Sabat R, Schulein R, et al: TIRC7 inhibits T cell proliferation by modulation of CTLA-4 expression. J Immunol 177: 6833-6841, 2006.

17. Frischer JM, Reindl M, Kunz B, Berger T, Schmidt S, Milford EL, Knosp E, Lassmann H and Utku N: TIRC7 and HLA-DR axis contributes to inflammation in multiple sclerosis. Mult Scler 20: 1171-1181, 2014.

18. Zhu F, Qiao J, Chen W, Pan B, Wu QY, Cao J, Sang W, Yan ZL, Zeng LY, Li ZY and Xu KL: Increased expression of T cell immune response cDNA 7 in patients with acute graft-versus-host disease. Ann Hematol 94: 1025-1032, 2015.

19. Utku N, Boerner A, Tomschegg A, Bennai-Sanfourche F, Bulwi GC, Heinemann T, Loehler J, Blumberg RS and Volk HD: TIRC7 deficiency causes in vitro and in vivo augmentation of $\mathrm{T}$ and $\mathrm{B}$ cell activation and cytokine response. J Immunol 173: 2342-2352, 2004.

20. Li J, Semple K, Suh WK, Liu C, Chen F, Blazar BR and Yu XZ: Roles of CD28, CTLA4, and inducible costimulator in acute graft-versus-host disease in mice. Biol Blood Marrow Transplant 17: 962-969, 2011.

21. Chen W, Li M, Li Z, Yan Z, Cheng H, Pan B, Cao J, Chen C, Zeng $\mathrm{L}$ and $\mathrm{Xu} \mathrm{K}$ : CXCR4-transduced mesenchymal stem cells protect mice against graft-versus-host disease. Immunol Lett 143: 161-169, 2012.

22. Zhao K, Zhao D, Huang D, Song X, Chen C, Pan B, Wu Q, Cao J, Yao Y, Zeng L and Xu K: The identification and characteristics of IL-22-producing T cells in acute graft-versus-host disease following allogeneic bone marrow transplantation. Immunobiology 218: 1505-1513, 2013.

23. Zhu S, Shi P, Lv C, Li H, Pan B, Chen W, Zhao K, Yan Z, Chen C, Loake GJ, et al: Loss of NLRP3 function alleviates murine hepatic graft-versus-host disease. Biol Blood Marrow Transplant 24: 2409-2417, 2018.

24. Blazar BR, Taylor PA, McElmurry R, Tian L, Panoskaltsis-Mortari A, Lam S, Lees C, Waldschmidt T and Vallera DA: Engraftment of severe combined immune deficient mice receiving allogeneic bone marrow via In utero or postnatal transfer. Blood 92: 3949-3959, 1998. 
25. Kaplan DH, Anderson BE, McNiff JM, Jain D, Shlomchik MJ and Shlomchik WD: Target antigens determine graft-versus-host disease phenotype. J Immunol 173: 5467-5475, 2004.

26. Qian L, Wu Z and Shen J: Advances in the treatment of acute graft-versus-host disease. J Cell Mol Med 17: 966-975, 2013.

27. Alegre ML, Frauwirth KA and Thompson CB: T-cell regulation by CD28 and CTLA-4. Nat Rev Immunol 1: 220-228, 2001.

28. Zheng J, Liu Y, Liu Y, Liu M, Xiang Z, Lam KT, Lewis DB, Lau YL and Tu W: Human $\mathrm{CD}^{+}$regulatory $\mathrm{T}$ cells inhibit GVHD and preserve general immunity in humanized mice. Sci Transl Med 5: 168ra9, 2013

29. Yoo JS, Lee YJ, Yoon JW, Hyunget KE and Hwang KW: CTLA-4-Tg/CD-28-KO mice exhibit reduced t cell proliferation in vivo compared to $\mathrm{CD}-28-\mathrm{KO}$ mice in a graft-versus-host disease model. Korean J Physiol Pharmacol 16: 349-353, 2012.

30. Zhu F, Qiao J, Zhong XM, Wu QY, Chen W, Yao Y, Niu MS, Fu CL, Zeng LY, Li ZY and Xu KL: Antithymocyte globulin combined with cyclosporine A down-regulates T helper 1 cells by modulating $\mathrm{T}$ cell immune response cDNA 7 in aplastic anemia. Med Oncol 32: 197, 2015.
31. Tamura A, Milford EL and Utku N: TIRC7 pathway as a target for preventing allograft rejection. Drug News Perspect 18 103-108, 2005.

32. Hwang KW, Sweatt WB, Brown IE, Blank C, Gajewski TF, Bluestone JA and Alegre ML: Cutting edge: Targeted ligation of CTLA-4 in vivo by membrane-bound anti-CTLA-4 antibody prevents rejection of allogeneic cells. J Immunol 169: 633-637, 2002.

33. Krummel MF, Sullivan TJ and Allison JP: Superantigen responses and co-stimulation: CD28 and CTLA-4 have opposing effects on $\mathrm{T}$ cell expansion in vitro and in vivo. Int Immunol 8: 519-523, 1996.

This work is licensed under a Creative Commons Attribution-NonCommercial-NoDerivatives 4.0 International (CC BY-NC-ND 4.0) License. 